\title{
Strain Localization Above the Yielding Point in Cyclically Deformed Glasses
}

\author{
Anshul D. S. Parmar, ${ }^{1,2}$ Saurabh Kumar, ${ }^{1}$ and Srikanth Sastry ${ }^{1, *}$ \\ ${ }^{1}$ Theoretical Sciences Unit, Jawaharlal Nehru Centre for Advanced Scientific Research, \\ Bengaluru, India \\ ${ }^{2}$ Tata Institute of Fundamental Research, Serilingampally Mandal, \\ Ranga Reddy District, Hyderabad, India
}

(Received 6 July 2018; revised manuscript received 24 January 2019; published 26 April 2019)

\begin{abstract}
We study the yielding behavior of a model glass under cyclic athermal quasistatic deformation and at finite rate and temperature, computationally, and show that yielding is characterized by the discontinuous appearance of shear bands, whose width is about ten particle diameters at their initiation, in which the strain gets localized. Strain localization is accompanied by a corresponding change in the energies and a decrease in the density in the shear band. We show that the glass remains well annealed outside the shear band, whereas the energies correspond to the highest possible energy minima at the given density within the shear band. Diffusive motion of particles characterizing the yielded state are confined to the shear bands, whose mean positions display movement over repeated cycles. Outside the shear band, particle motions are subdiffusive but remain finite. Despite the discontinuous nature of their appearance, shear bands are reversible in the sense that a reduction in the amplitude of cyclic deformation to values below yielding leads to the healing and disappearance of the shear bands.
\end{abstract}

DOI: 10.1103/PhysRevX.9.021018

\section{INTRODUCTION}

The mechanical response of amorphous solids to applied stresses is of obvious importance in characterizing their behavior. Beyond the elastic regime at small applied stresses, such response is characterized by plastic deformations, and beyond yielding, by flow. Considerations associated with yielding are of relevance to a wide range of phenomena, from irreversible deformation and failure in atomic and molecular glasses, such as metallic glasses, to the complex rheology of soft materials such as foams, emulsions, colloidal suspensions, and granular matter [1-5]. Although yielding and flow may apparently be continuous and homogeneous for some yield stress fluids, it is a sharp, discontinuous event at the other end of the spectrum, as in brittle failure, characterized by localization of strain and the formation of shear bands. Systems and questions of interest range from the mechanical properties of nanostructures to large-scale phenomena such as mudslides and earthquakes [6-9]. Viewed as a nonequilibrium transition in a driven system, the phenomena associated with yielding have in recent years been investigated in a large number of studies experimentally, through

\footnotetext{
*sastry@jncasr.ac.in
}

Published by the American Physical Society under the terms of the Creative Commons Attribution 4.0 International license. Further distribution of this work must maintain attribution to the author(s) and the published article's title, journal citation, and DOI.
Subject Areasxx: Condensed Matter Physics, Soft Matter, Statistical Physics computer simulations, and the analysis of elastoplastic and other models [10-36]. Many studies have focused on the anisotropic interactions arising between localized plastic events or STZs [21,37-42] and, in particular, how they may influence strain localization. Yielding has been analyzed through the application of STZ theory $[3,18]$, described as a critical transition in analogy with depinning of manifolds in random media [17,23], discontinuous transition associated with a spinodal [32-36,43-47], etc., and the relationship between these descriptions is a subject of ongoing investigation (see, e.g., Refs. [35,36]). The role of the degree of annealing in determining the nature of the yielding transition, and in the formation and character of shear bands, has increasingly been appreciated $[13,31,35,36,48]$. A particular situation in which the role of annealing becomes manifest is when an amorphous solid is subjected to oscillatory shear deformation [8,24,26,31,49-53], a protocol that also reveals transitions to irreversible states at large driving in other soft matter systems, such as non-Brownian suspensions [14]. Under oscillatory or cyclic deformation of a glass, an increasing degree of annealing is observed as the amplitude of deformation is increased [31,49] (manifested by a decrease in energy) until the yielding strain is reached. Beyond the yielding strain, as described in detail below, the system yields through the formation of a shear band, within which the strain becomes largely localized.

The overall energy of the glass increases from the yielding strain onwards. The width of the shear band increases with an increase in the applied strain amplitude, 
but at any given amplitude, it reaches a steady-state value for large numbers of cycles of deformation. However, since the system becomes inhomogeneous, one may inquire about the state of the glass within and outside the shear band. We investigate these changes in the present work, employing athermal quasistatic (AQS) deformation of a model glass, and show that the mechanical response of a cyclically deformed glass simultaneously displays features of aging or annealing outside the shear band, and of rejuvenation within. Results from athermal quasistatic deformation are subject to the criticism that the resulting description of mechanical response of amorphous solids is unrealistic, as the method neglects inertial and thermal effects, and thus, dynamic relaxation mechanisms that may be of relevance to the phenomenon under study (see, e.g., Ref. [54]). Here, we show convincingly that this is not the case for our results regarding yielding. Finite-temperature and shear-rate simulations display behavior that is qualitatively indistinguishable from the behavior observed in AQS simulations.

In Sec. II, we describe the model we study and the methods employed. In Sec. III, we give our results. In Sec. IV, we discuss the conclusions and implications of the work presented.

\section{MODEL AND METHODS}

We simulate the Kob-Andersen binary $(80: 20)$ mixture of 64000 Lennard Jones particles at a reduced density of $\rho=1.2$. Quadratic corrections are used to make the force and the potential energy continuous at the cutoff $r_{c \alpha \beta}\left(=2.5 \sigma_{\alpha \beta}\right)$. The pairwise interactions are defined as

$$
\begin{array}{rlr}
U_{\alpha \beta}(r)= & 4 \epsilon_{\alpha \beta}\left[\left(\frac{\sigma_{\alpha \beta}}{r}\right)^{12}-\left(\frac{\sigma_{\alpha \beta}}{r}\right)^{6}\right] \\
& +4 \epsilon_{\alpha \beta}\left[c_{0 \alpha \beta}+c_{2 \alpha \beta}\left(\frac{r}{\sigma_{\alpha \beta}}\right)^{2}\right], \quad & \\
= & 0, \quad \text { otherwise. }
\end{array}
$$

Here, indices $\alpha, \beta \in\{A, B\}$ refer to particle type, $c_{0 \alpha \beta}$ and $c_{2 \alpha \beta}$ are chosen to ensure that the potential and its derivative at $r_{c \alpha \beta}$ vanish at the cutoff, and interaction parameters, defined with respect to the particles of type " $A$," are $\epsilon_{A B} / \epsilon_{A A}=1.5, \quad \epsilon_{B B} / \epsilon_{A A}=0.5, \quad \sigma_{A B} / \sigma_{A A}=0.80, \quad$ and $\sigma_{B B} / \sigma_{A A}=0.88$. Energy and length are expressed throughout in units of $\epsilon_{A A}$ and $\sigma_{A A}$, respectively. Initial samples are generated by equilibrating the system at a high temperature $T=1$ (in reduced units) using the Nosé-Hoover thermostat. We perform cyclic deformation for the most part over a range of amplitudes $\gamma_{\max }$ above the yield strain amplitude $\left(\gamma_{y} \approx 0.07\right.$, see Refs. [31,49]), from 0.07 to 0.11 . We consider as starting configurations either the energy minimum structures (inherent structures) obtained from minimizing high-temperature liquid configurations $(T=1)$, or previously cyclically deformed configurations at $\gamma_{\max }=$ 0.07 and 0.08 , which have reached a steady state. The shear deformation is carried out using the AQS protocol, wherein each deformation step by a small strain increment is followed by energy minimization using the conjugate gradient method. Samples are subjected to volume preserving shear along the $x z$ plane by incrementing strain by small steps of $d \gamma_{x z}$ (here, $2 \times 10^{-4}$ ) via the coordinate transformation of $x^{\prime}=x+z d \gamma_{x z}, y^{\prime}=y, z^{\prime}=z$. In addition to extensive AQS simulations, we study the yielding behavior at finite temperatures and strain rate. The trajectories are evolved via the SLLOD algorithm [55]. The applied strain $\gamma(t)$ is of the form $\gamma(t)=\gamma_{\max } \sin (\omega t)$, where $\omega$ is the frequency and $\gamma_{\max }$ is the amplitude of the strain. The strain rates $\dot{\gamma}$ are reported at the initial time of each cycle, i.e., $\dot{\gamma}=\gamma_{\max } \omega$. We deform the box at a small strain rate $\dot{\gamma}(t=0)=5 \times 10^{-5}$ and at temperature $T=0.1$ and $T=.001$, and study the yielding behavior. We observe the formation of shear bands and estimate the yielding strain amplitude in each case. The AQS and SLLOD simulations are performed using LAMMPS [56]. Lees-Edwards periodic boundary conditions are employed in both the energy calculation and minimization. We perform cyclic shear deformation $\left(0 \rightarrow \gamma_{\max } \rightarrow 0 \rightarrow-\gamma_{\max } \rightarrow 0\right)$ repeatedly, until a steady state is achieved. As previously described $[31,49]$, the number of cycles needed to reach a steady state rises steeply as $\gamma_{y}$ is approached, but we do not discuss this feature further here. For all the strain amplitudes above $\gamma_{y}$ studied, the steady-state configurations display shear bands.

To characterize the shear bands, we divide the configurations into slabs along the shear direction and compute (a) the mean-squared displacement $[\mathcal{M S D}(z)]$ between particle positions in a slab centered at $z$ at strain $\gamma=0$ separated by a full cycle of strain, and (b) the average energies of particles in each slab, $U(z)$, at the end of each cycle (i.e., we consider stroboscopic configurations). For a given cycle $i$, the MSD for the $z$ th slab is written as $\operatorname{MSD}^{(i)}(z)=\sum_{j=1}^{n_{i}^{z}}\left(\mathbf{r}_{i+1, j}^{z}-\mathbf{r}_{i, j}^{z}\right)^{2} / n_{i}^{z}$, where $n_{i}^{z}$ and $\left\{\mathbf{r}_{i}^{z}\right\}$ represent the number of particles and their positions in the $z$ th slab of the stroboscopic configuration in the $i$ th cycle, respectively. Similarly, the slabwise energy can be defined as $U^{(i)}(z, i)=\sum_{j=1}^{n_{i}^{z}} u_{i, j}^{z} / 2 n_{i}^{z}$, where $u_{i, j}^{z}$ is the interaction energy of the $j$ th particle in the $z$ th slab. The cycle index $i$ is not indicated unless necessary. The profile of $\operatorname{MSD}(z)$ is found to be well described by a Gaussian, $\operatorname{MSD}(z)=$ $\mathrm{MSD}^{o} \exp \left(-(z-\langle z\rangle)^{2} / 2 \sigma^{2}\right)$, where $\langle z\rangle$ and $\sigma$ represent the mean position and width of the shear band, respectively. In order to characterize properties within and outside the shear band, we compute various partial (per particle) averages (other than the slabwise averages defined above), which we define here with the interaction energy as an example: Partial averages are computed for the center of the shear band $\left[U_{\mathrm{SB}}(\sigma)\right]$, most of the shear band (within $3 \sigma$ ) $\left[U_{\mathrm{SB}}(3 \sigma)\right]$, the rest of the system (outside $\left.3 \sigma\right)\left(U_{\text {rest }}\right)$, the slab of thickness $\sigma$ farthest from the center of the shear band $\left[U^{\prime}(\sigma)\right]$, and the global average value $(U)$. In addition to the 
MSD and energy $U$, we also compute the average displacement of particles per cycle, defined (for the full system) as $\Delta r^{(i)}=\sum_{j=1}^{N}\left|\mathbf{r}_{i+1, j}-\mathbf{r}_{i, j}\right| / N$, for cycle $i$, and the mean-squared displacement with respect to a reference configuration $\mathbf{r}_{j}^{0}$ for the $A$ particles as $\left\langle r_{A}^{2}\right\rangle^{(i)}=$ $\sum_{j=1}^{N_{A}}\left(\mathbf{r}_{i, j}-\mathbf{r}_{j}^{0}\right)^{2} / N_{A}$.

\section{RESULTS}

In Fig. 1, we report the characterization of the shear band. We show configurations from the steady state for a given strain amplitude, $\gamma_{\max }=0.09$. The color map is

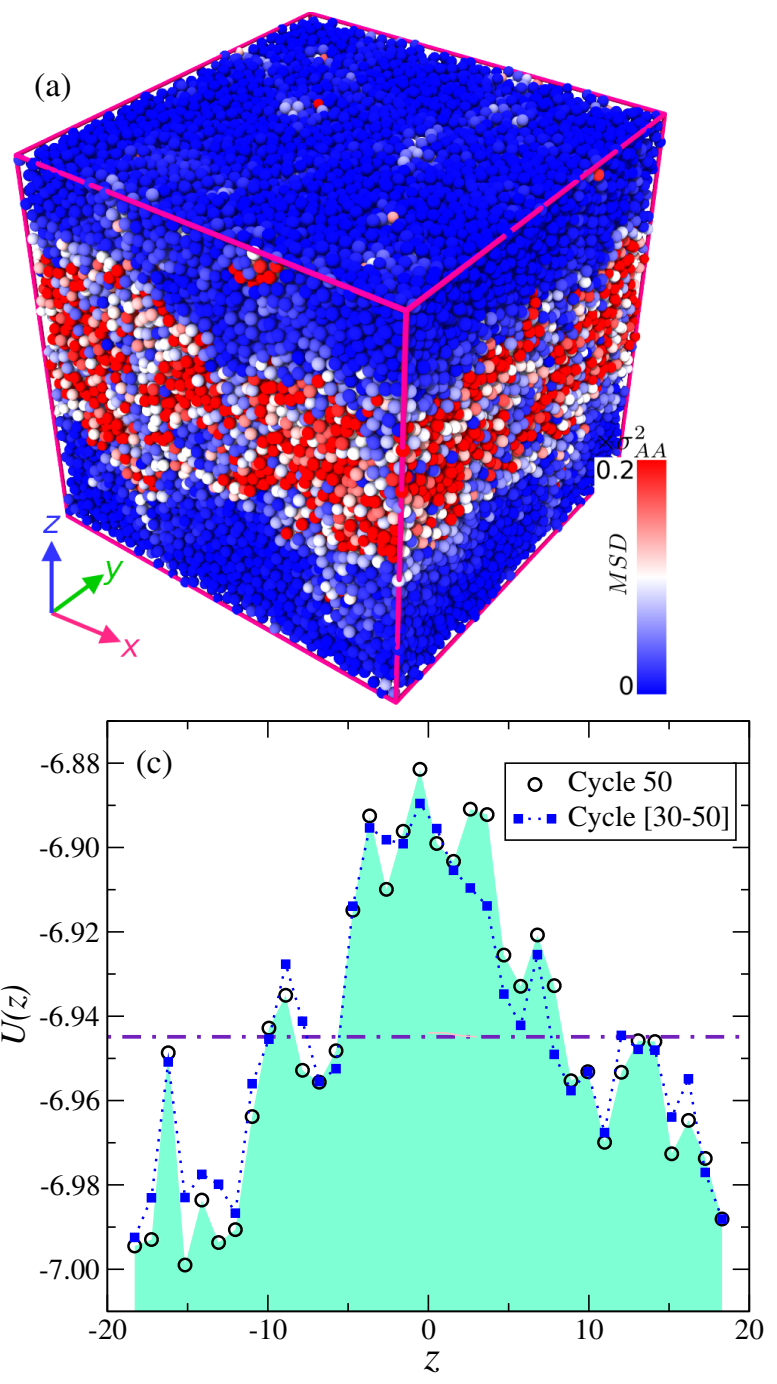

based on the mean-squared displacement within a slab between two consecutive strain cycles. Particles shown in red move more than $0.2 \sigma_{A A}^{2}$ (MSD values roughly within $\langle z\rangle \pm \sigma)$. Figure 1(b) shows the $\operatorname{MSD}(z)$ profile of the steady-state configuration, which clearly shows the existence of strain localization or a shear band. The existence of a shear band also gets reflected in the energy profile of particles vs the $z$ coordinate [see Fig. 1(c)]; particles corresponding to the band are likely to have higher energy compared to the mean potential energy of the system. Figure 1(d) shows the deviation from the affine strain in the displacement profile between the stroboscopic configuration $(\gamma=0)$
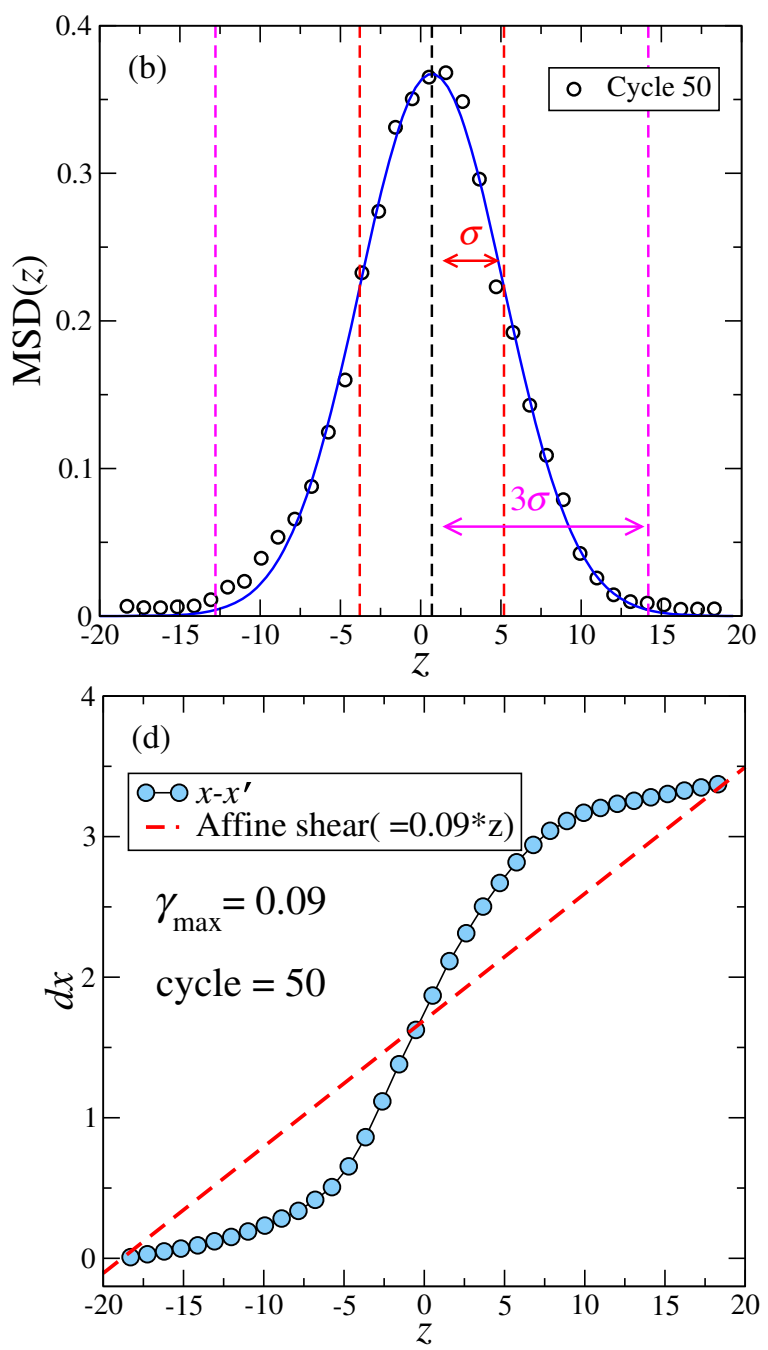

FIG. 1. (a) Snapshot of a configuration from the steady state for the strain amplitude $\gamma_{\max }=0.09$, with the color of the particle indicating the mean-squared displacement (MSD) over a strain cycle. Highly mobile particles (MSD $>0.2 \sigma_{A A}^{2}$ ) are colored in red, whereas particles in blue move considerably less. This snapshot shows that particle displacements are highly spatially correlated, forming a shear band. (b) $\operatorname{MSD}(z)$ is shown as a function of the coordinate $z$ in the shear direction, along with a Gaussian fit. For our analysis, we consider most mobile particles (within a width of $1 \sigma$ ) and most of the particles (within $3 \sigma$ ) in the shear band. (c) The potential energy of mobile particles is seen to be higher than the mean potential energy represented by a horizontal line. The data presented by black circles correspond to the 50th cycle of strain, whereas data shown in blue boxes are averaged over 20 cycles (from 30 to 50). (d) We plot the displacement profile $\left[d x=x(\gamma=0)-x\left(\gamma_{\max }\right)\right]$, as a function of the coordinate in the gradient direction. The deviation from the affine field suggests the presence of strain localization. 

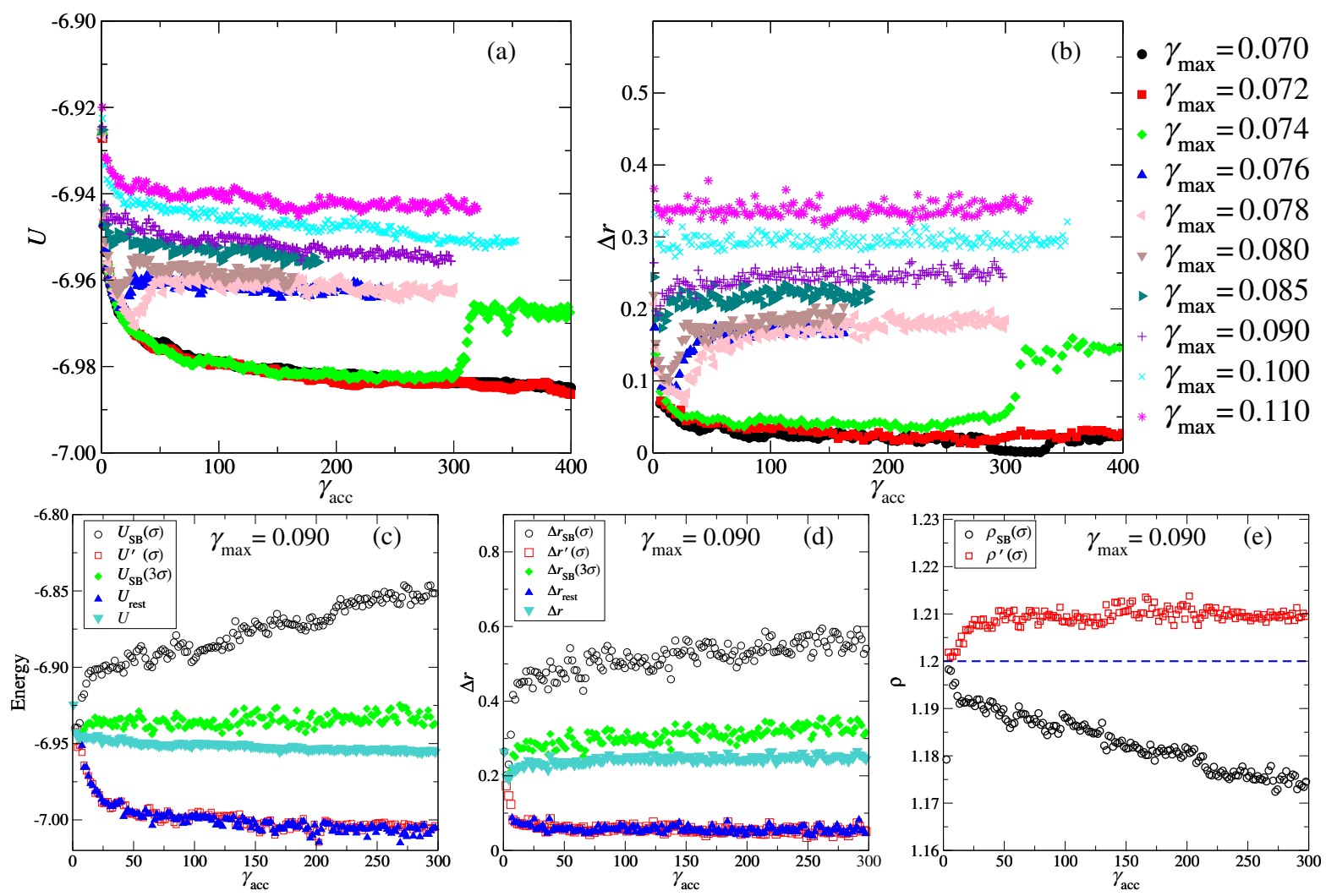

FIG. 2. (a) The potential energy per particle $(U)$ of stroboscopic configurations and (b) averaged particle displacement $(\Delta r)$ after one deformation cycle as a function of accumulated strain $\left(\gamma_{\text {acc }}\right)$ for various strain amplitude. For $\gamma_{\max }<\gamma_{y}$, the system remains annealed, and the corresponding single-particle displacements are small. The nonmonotonic behavior at higher amplitudes arises from shear banding. (c) The potential energy and (d) averaged particle displacement for (i) the center of the shear band [ $\left.U_{\mathrm{SB}}(\sigma) \& \Delta r_{\mathrm{SB}}(\sigma)\right]$, (ii) the slab farthest away $\left[U^{\prime}(\sigma) \& \Delta r^{\prime}(\sigma)\right]$, (iii) most of the shear band $\left[U_{\mathrm{SB}}(3 \sigma) \& \Delta r_{\mathrm{SB}}(3 \sigma)\right]$, (iv) the rest of the system ( $\left.U_{\text {rest }} \& \Delta r_{\text {rest }}\right)$, and (v) the entire system $(U \& \Delta r)$ as a function of accumulated strain. The system outside the shear band remains annealed even for large strain amplitudes above the yielding amplitude. (e) The local density of the shear band decreases compared with the mean density of the system.

and the corresponding sheared sample at strain $\gamma_{\max }=0.09$ indicating the localization of the strain in the steady states.

We next consider a detailed analysis of the energies and displacements as a function of accumulated strain $\gamma_{\text {acc }} \equiv$ $N_{\text {cycles }} \times 4 \gamma_{\max }$ for a range of strain amplitudes spanning the yield strain amplitude. In Fig. 2, the potential energy $U$, average displacement per cycle $\Delta r$ and the density $\rho$ of the full system, as well as various subvolumes (within and outside the shear band), are shown against $\gamma_{\text {acc }}$. The initial sample corresponds, in each case, to an inherent structure obtained from liquid configurations at high temperature $(T=1)$, roughly corresponding to the highest inherent structure energy at fixed density, often referred to as the top of the landscape. For the smaller amplitudes, the energy $U$ and displacements $\Delta r$ decrease monotonically, indicating considerable annealing, as previously observed [31]. For larger amplitudes, the variation with $\gamma_{\text {acc }}$ is nonmonotonic, and the sharp upward changes (seen most markedly for $\left.\gamma_{\max }=0.074\right)$ indicate the spontaneous onset of shear banding (see Supplemental Material [57] for a movie that demonstrates shear-band formation starting with a homogeneous state). Energy and $\Delta r$ values shown as averages within and outside the shear band for $\gamma_{\max }=0.09$ in Figs. 2(c) and 2(d) reveal that the upward changes arise within the shear band $\left[U_{\mathrm{SB}}(3 \sigma)\right.$ and $U_{\mathrm{SB}}(\sigma), \Delta r_{\mathrm{SB}}(3 \sigma)$ and $\left.\Delta r_{\mathrm{SB}}(\sigma)\right]$, whereas outside $\left[U_{\mathrm{SB}}^{\prime}(\sigma), U_{\text {rest }}, \Delta r^{\prime}(\sigma), \Delta r_{\text {rest }}\right]$ continued annealing is revealed by the monotonic decrease of the energy and displacements. Figure 2(e) shows densities within and outside the shear band, displaying a densification outside the shear band, whereas within, the density shows significant reduction.

We next show, in Fig. 3(i) the mean potential energy, (ii) the width of the shear band, and (iii) the fraction of particles within the shear band. In Fig. 3(a), we show the mean potential energy of the total system as a function of strain amplitude, which changes discontinuously across the yielding amplitude. Such behavior has been described as rejuvenation $[31,49,58]$, but the energy values within and outside the shear band make it clear that while the part of the system within the shear band rejuvenates (attains higher energies), the rest of the system continues to anneal. 

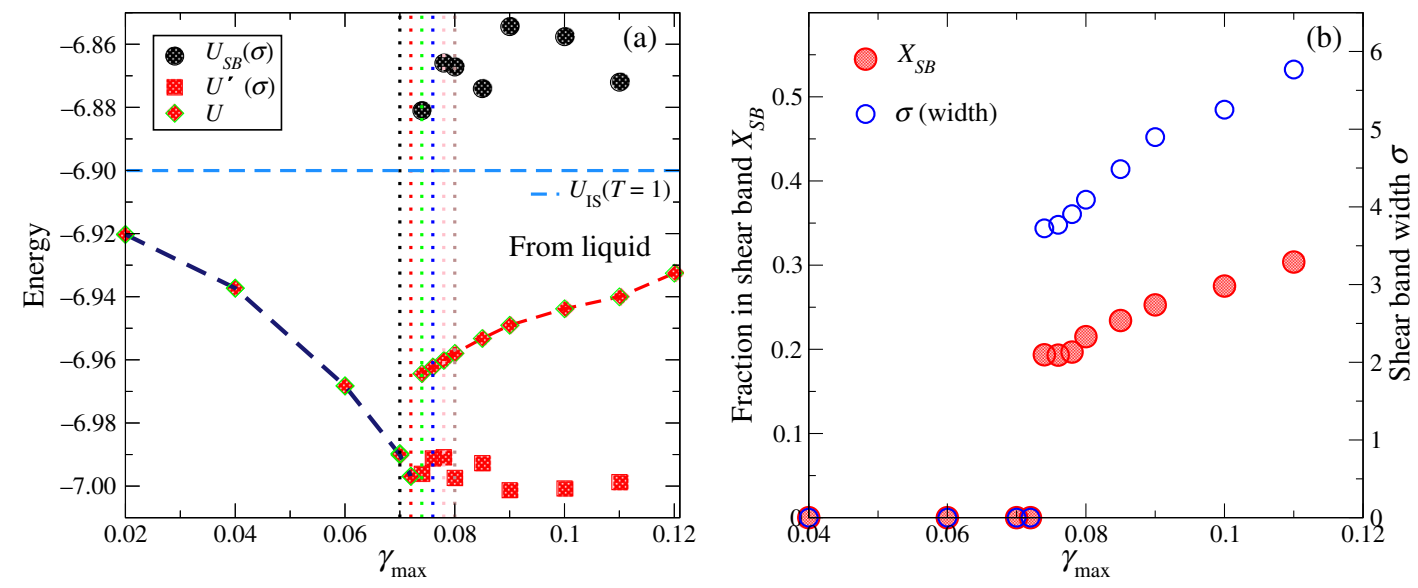

FIG. 3. (a) The mean energy of the system, shear band, and outside (farthest $\sigma$-wide slab) is plotted against strain amplitude. The mean energy changes discontinuously across the yielding transition. The system outside the shear band is increasingly better annealed for amplitudes even beyond the yield amplitude, whereas particles in the shear band have energies higher than the initial inherent structure energies, owing to a reduction in density. The region between the strain amplitude 0.07 to 0.08 is highlighted by equally spaced lines, with the interval of strain 0.002. (b) The thickness of band $(\sigma)$ and the fraction of particles $\left(X_{\mathrm{SB}}\right.$, within $\left.\sigma\right)$ of the shear band change from zero discontinuously to finite values at the yielding point.

The jump in the mean potential energy is coincident with the coming into existence of a shear band with finite width (with core width $2 \sigma \approx 7.5 \sigma_{A A}$ at the yielding strain). Figure $3(\mathrm{~b})$ shows the width of the shear band $(\sigma)$ and the fraction of particles within the shear band, underscoring the discontinuous nature of the transition.

Figure 4(a) shows the mean position $\langle z\rangle$ of the shear band for $\gamma_{\max }=0.090$. The mean position shows movement over distances comparable to the dimensions of the simulated system, lending credence to the characterization of the state of the system above yielding as ergodic [49], which may be doubted in the presence of shear banding. We calculate the mean-squared displacements $\left\langle r_{A}^{2}\right\rangle$ (for $A$ types of species) in the steady state for the entire system, $\sigma, 3 \sigma$, and the slab of thickness $\sigma$ farthest from the shear band, for a range of strain amplitudes. The inset in Fig. 4(a) shows the variation in the width of the shear with accumulated strain. Once the shear band is formed, the width of the band does not vary significantly over the long simulations, providing clear evidence of the stability of the shear band under cyclic shear deformation. As shown in Fig. 4(b), data for the entire system and the shear band show diffusive behavior, whereas for the slab of thickness $\sigma$ farthest from the shear band (annealed region), the behavior is subdiffusive. The diffusion coefficients $\left(D_{A}\right)$ estimated from the fit function, $\left\langle r_{A}^{2}\right\rangle\left(\gamma_{\text {acc }}\right)=$ $D_{A} \gamma_{\text {acc }}$, and shown in Fig. 4(c) indicate that the diffusion coefficients change discontinuously from zero to a finite value across the yielding transition, consistently with the findings in Ref. [52]. Such discontinuous behavior is also seen in the behavior of $\langle\Delta r\rangle$, for which [Fig. 4(d)] a decomposition into values within and outside the shear band shows that the discontinuity arises from the emergence of the shear band.

Figure 5 shows results obtained when cyclic shear deformation is applied to differently prepared initial samples for a range of amplitudes above and below the yielding amplitude. In addition to the inherent structures obtained from the liquid at $T=1.0$, which we have discussed so far, we consider as initial configurations the steady-state configurations obtained at strain amplitude $\gamma_{\max }=0.08$ (which are shear banded) and at strain amplitude $\gamma_{\max }=0.07$ (well-annealed samples below the yielding point). Figures 5(a)-5(c) show results for an initially shear banded sample (prepared at $\gamma_{\max }=0.08$ ) subjected to cyclic deformation for a range of amplitudes above and below the yielding amplitude. The energies as a function of accumulated strain $\gamma_{\text {acc }}$ are shown in Fig. 5(a). When $\gamma_{\max }$ is below the yielding amplitude, the shear band is gradually annealed out, and the system achieves energies that are lower than those obtained when inherent structures from the high-temperature liquid at $T=1$ are used as initial configurations [see Fig. 5(b)]. This result is clearly demonstrated in Fig. 5(c) through $\operatorname{MSD}(z)$ shown at different numbers of cycles, which reveal that the amplitude of the MSD gradually diminishes and becomes negligible. In other words, by performing cyclic deformation at an amplitude below yielding, even an initially shear banded state reaches a final state that is homogeneous and well annealed. On the other hand, when a sample annealed at $\gamma_{\max }=0.07$ is subjected to larger amplitude strain, it shear bands (for $\gamma_{\max } \geq 0.074$ ), as seen from the energies shown in Figs. 5(d) and 5(e), with the energies outside the shear band decreasing with increasing strain amplitude. The fraction of particles within the shear band and the thickness are independent of the initial sample [see Fig. 5(f)], demonstrating that a unique steady state is reached regardless of initial conditions, dependent only on the strain amplitude (more generally, parameters determining the cyclic deformation protocol), with the discontinuous appearance of shear bands in all cases. 

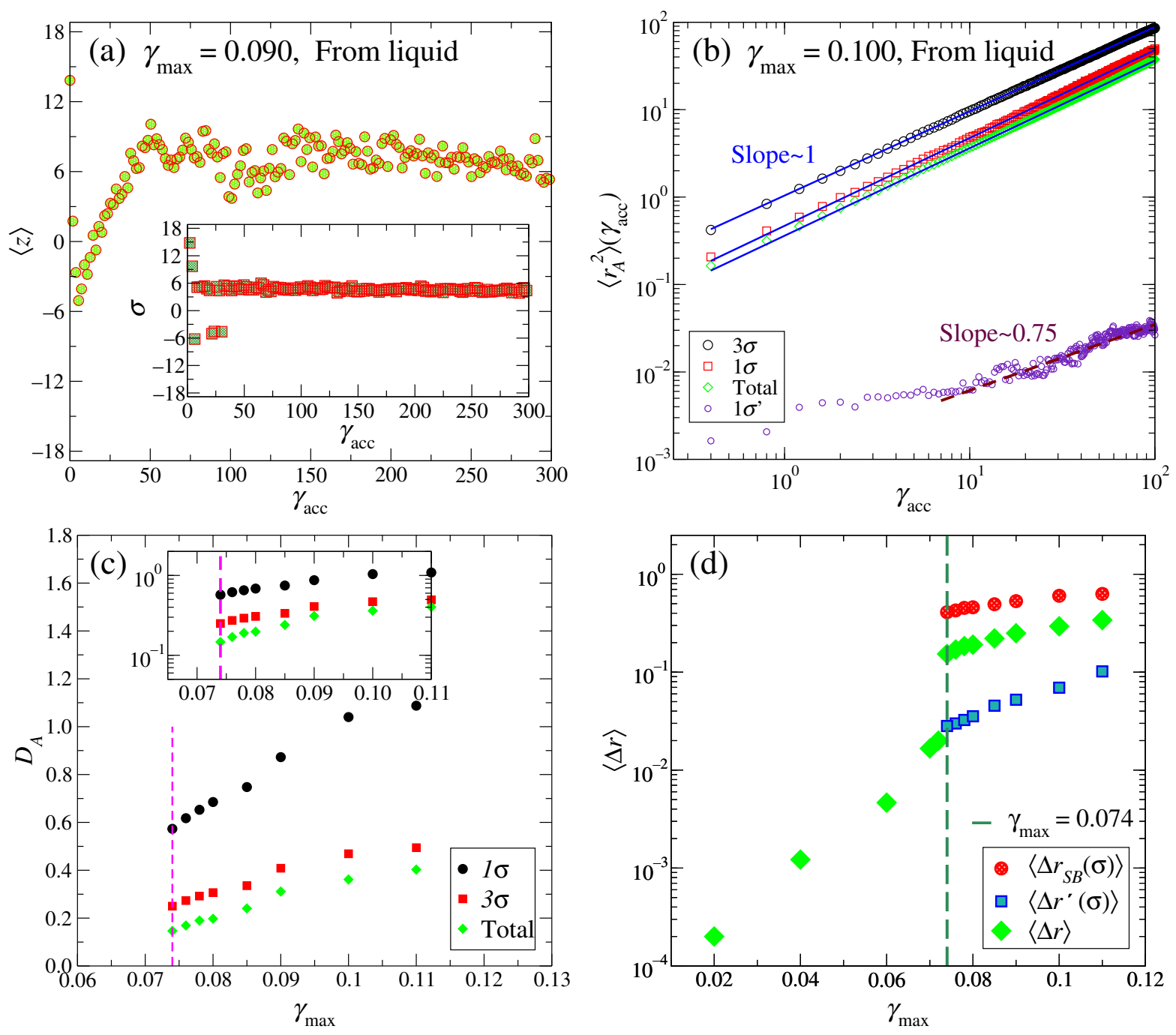

FIG. 4. (a) The mean position of the shear band, $\langle z\rangle$, showing large-scale variation with accumulated strain $\gamma_{\text {acc }}$. The inset shows the width " $\sigma$ " of the shear band with $\gamma_{\text {acc }}$, which does not change much over the entire simulation. (b) The mean-squared displacement of the " $A$ " type of particles for the full system. Particles within the shear band $(\sigma, 3 \sigma)$ show linear variation with $\gamma_{\text {acc }}$, whereas at the slab farthest away $\left(1 \sigma^{\prime}\right)$, it is subdiffusive. (c) The diffusion coefficient of particles of type $A$ for the center of the shear band $(\sigma)$, most of the shear band ( $3 \sigma)$, and of the total system, showing discontinuous change across the yielding point. The inset shows the same data on a semilog scale. (d) The steady-state values of $\Delta r$ for the total system $(\langle\Delta r\rangle)$, shear band $\left[\left\langle\Delta r_{\mathrm{SB}}(\sigma)\right\rangle\right]$, and the slab of thickness $\sigma$ farthest away from the shear band $\left[\left\langle\Delta r^{\prime}(\sigma)\right\rangle\right]$, plotted against applied strain amplitudes. The $\langle\Delta r\rangle$ of the full system and the shear band change in a discontinuous manner across the yielding amplitude. Note that the $\Delta r$ values outside show continuous variation with values below the yielding amplitude.

We finally show, in Fig. 6, the behavior of glass configurations when sheared at finite temperatures and strain rates. We study two temperatures, $T=0.001$, and $T=0.1$, the latter temperature being a third of the previously estimated ideal glass transition temperature for the studied system. As in the case of AQS, for small amplitudes, we observe that the glasses are annealed to lower energies, with the degree of annealing, as well as the number of cycles needed to reach the steady-state values, increasing with increasing strain amplitude. We perform up to 400 cycles of deformation for $T=0.001$, and 600 cycles for $T=0.1$. A discontinuous jump in the energies is observed above a strain amplitude, which we identify as the yielding strain amplitude $\gamma_{y}$, which we find to be $\gamma_{y}=0.05$ for $T=0.1$ and $\gamma_{y}=0.07$ for $T=0.001$. Figures 6(a) and 6(b) show that the energies (U) vs $\gamma_{\max }$ decrease with increasing $\gamma_{\max }$ below $\gamma_{y}$ and increase above the yield amplitude for both temperatures, $T=0.1$ and $T=0.001$. Since the energies continue to decrease for the number of cycles studied for amplitudes below yielding, the asymptomatic energies $[U(\infty)]$ are obtained by fitting to a stretched exponential function of the form $U(n)=$ $(U(0)-U(\infty)) e^{-\left(n / n^{*}\right)^{\beta}}+U(\infty)$, where $n^{*}$ is the number of cycles to reach a steady state and $U(\infty)$ is the limiting value of the energy. Above yielding, the steady-state values are reached within the length of our simulations and are reported. The snapshots in Figs. 6(a) and 6(b) show that the yielding is associated with the discontinuous 

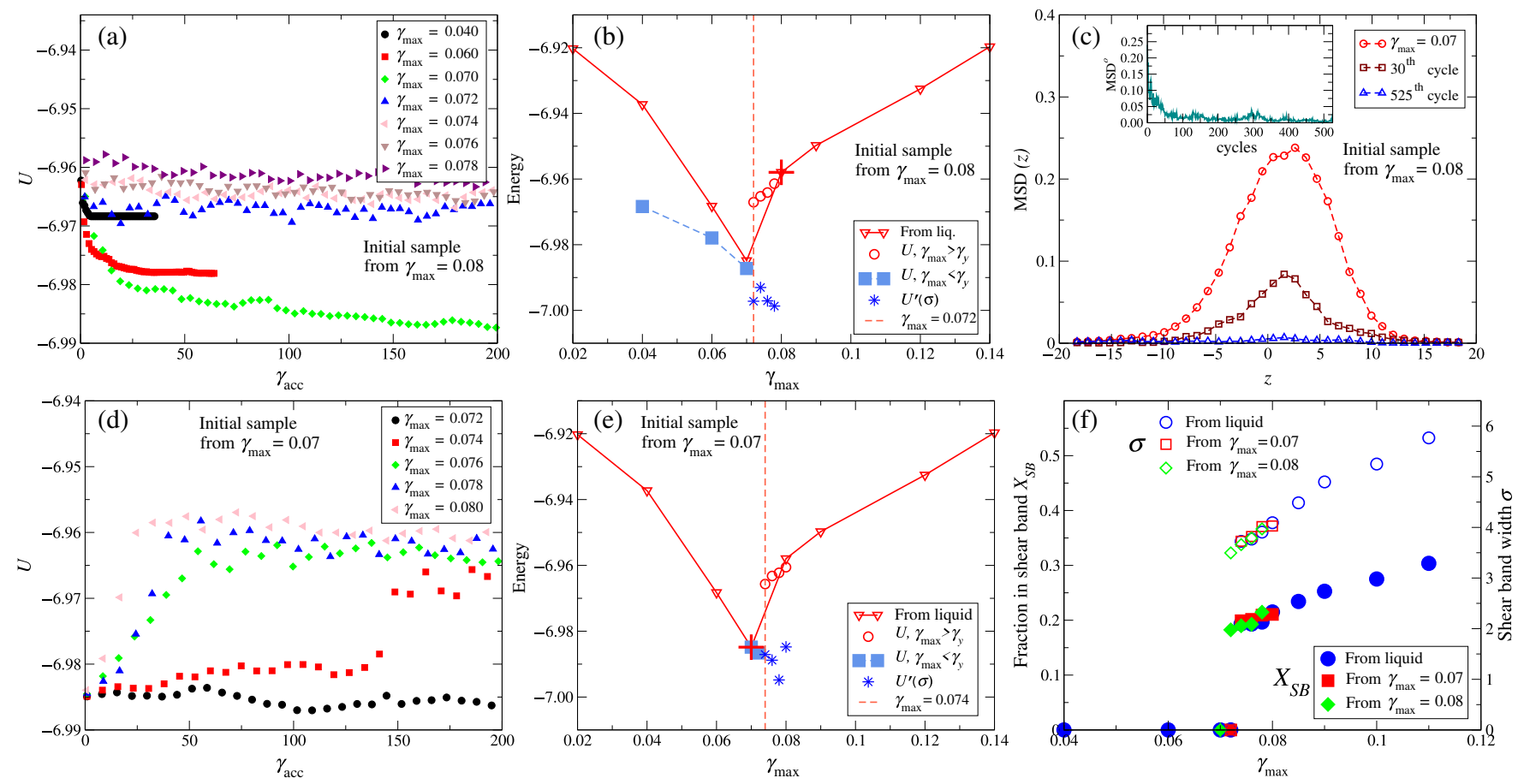

FIG. 5. (a,d) Initial samples from the steady state from $\gamma_{\max }=0.08$ [(a), shear banded] and from $\gamma_{\max }=0.07$ [(b), annealed sample] are deformed for a range of amplitudes across the yielding point. The potential energy per particle $(U)$ of stroboscopic configurations as a function of accumulated strain $\left(\gamma_{\text {acc }}\right)$ for various strain amplitude is shown. (b,e) Average energies for below and above the yield amplitude of the sample with initial configurations from steady states at strain amplitudes 0.08 and 0.07 . Below the yield amplitude $\left(<\gamma_{y}\right)$, the initial shear band anneals out [e.g., sample from 0.08 amplitude, as seen in (c)], leading to energies below those obtained with inherent structures from the high-temperature liquid at $T=1$ as initial configurations (red triangles shown as reference). Above yielding, energies within and outside the shear band are different. (c) $\operatorname{MSD}(z)$ along the $z$ direction at different cycles showing the annealing out of the shear band. The inset shows the amplitude $\mathrm{MSD}^{o}$ for the shear band vs accumulated strain. (f) The fraction of particles in the shear band and the width of the shear band remains independent of the initial sample.
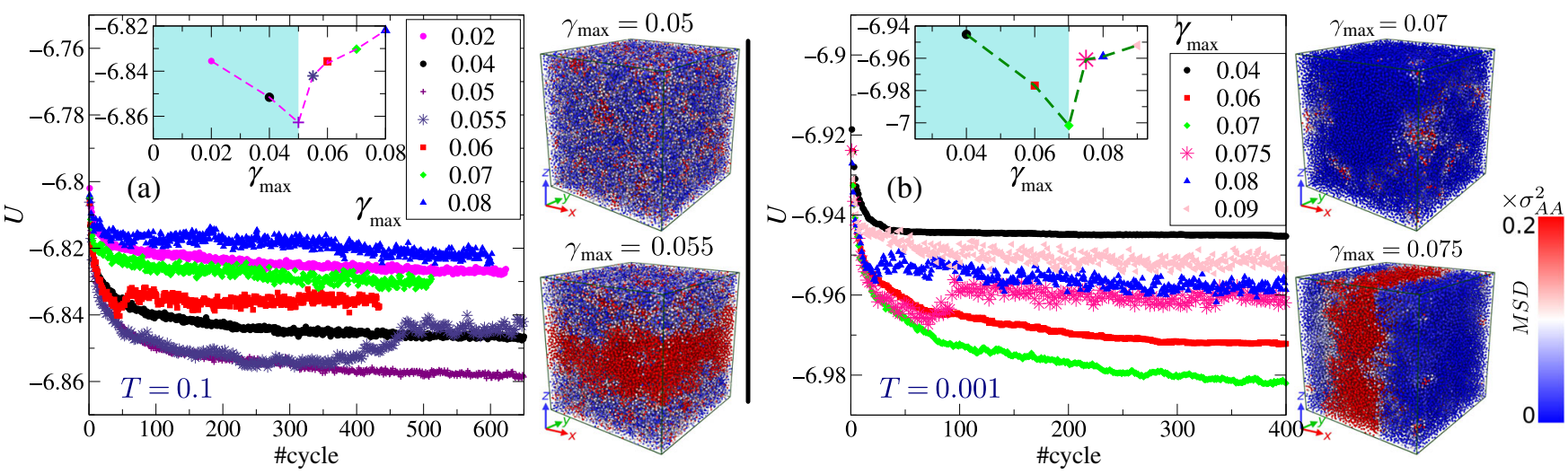

FIG. 6. (a,b) Evolution of the potential energy as a function of the number of strain cycles at a fixed temperature (a) $T=0.1$ and (b) $T=0.001$ at the shear rate $\left(\dot{\gamma}=5 \times 10^{-5}\right)$ implemented via SLLOD [55]. Following earlier analysis [31], we identify the location of minimum energy to be the yield strain amplitude $\gamma_{y}$, and we find $\gamma_{y}=0.05$ (for $T=0.1$ ) and $\gamma_{y}=0.07$ (for $T=0.001$ ) to within the precision of our sampling of strain amplitudes. Insets show the asymptomatic $\left(\gamma_{\max }<\gamma_{y}\right)$ and steady-state $\left(\gamma_{\max }>\gamma_{y}\right)$ values of the potential energy. (a) Snapshots are shown of configurations from the 600th cycle for the strain amplitude $\gamma_{\max }=0.05$ and $\gamma_{\max }=0.055$, $T=0.1$. (b) Snapshots are shown of configurations from the 400th cycle for the strain amplitude $\gamma_{\max }=0.07$ and $\gamma_{\max }=0.075$, $T=0.001$. The color of the particle is based on the MSD over a strain cycle. These snapshots reveal that, above the yielding strain amplitude, particle displacements are highly spatially correlated, very similarly to findings from AQS simulations. 
appearance of a shear band. For the smaller strain amplitudes $\left(\leq \gamma_{y}\right)$, the particle displacements during a cycle are not spatially correlated, but for amplitudes above the yield $\left(>\gamma_{y}\right)$, they are, which leads to the formation of shear bands. These results are indistinguishable from the ones from AQS simulations in broad qualitative features. Some details, such as the temperature dependence of $\gamma_{y}$, vary and are highly interesting to investigate in future work.

\section{DISCUSSION}

In summary, we have investigated strain localization and formation of shear bands accompanying yielding in glasses subjected to cyclic shear deformation, both using the athermal quasistatical protocol and at finite shear rate and temperature. The qualitative features of the yielding and strain localization obtained from AQS, and finitetemperature, shear-rate simulations are indistinguishable. However, we find that the location of the yielding transition is temperature dependent. The strain rate and temperature dependence of the yielding transition are important issues, which will be addressed in future work in more detail.

Shear bands with a thickness of several atomic diameters emerge discontinuously when the yielding amplitude of deformation is exceeded, consistently with, and illustrating, the descriptions of yielding as a discontinuous transition for well-annealed glasses [13,29,31-36]. Under cyclic deformation at small amplitudes, the energy of the homogeneous glasses evolves towards lower energies with repeated cycles; i.e., it anneals. Yielding constitutes a point of instability, where it is no longer possible to anneal the system homogeneously, and part of the system attains higher energy structures, which carry the excess strain that cannot be accommodated by increased annealing of the glasses. Even at amplitudes beyond yield, a large fraction of the system continues to exhibit annealing, a condition that has been analyzed to be associated with the formation of shear bands. Thus, shear-banded structures simultaneously show features of rejuvenation (inside the shear band) and annealing or aging (outside the shear band). Homogeneous, or shear-banded, structures result as stable steady-state structures that are governed by the conditions of cyclic driving, regardless of the initial state from which they are prepared. We thus find the remarkable feature that initially shear-banded configurations, when subjected to cyclic deformation at amplitudes smaller than the yield amplitude $\gamma_{y}$, attain a homogeneous state, with the healing out of the shear bands.

Experiments employing cyclic deformation to probe yielding in soft glasses (emulsions [24] and colloidal suspensions [25,59]) observe a sudden onset of yielding, which is consistent with our observations. However, these works appear to have different statements to make regarding plastic arrangements accompanying the yielding transition. In Refs. [24,25], based on estimates of a correlation length for rearranging particles (or sizes of clusters of rearranging particles), it is argued that such a correlation length is maximum at the yielding transition. On the other hand, Ref. [59] reports that, while there are virtually no irreversible rearrangements below yielding, a substantial population of plastic arrangements is found above. The observations in Ref. [59] are consistent with our own observations, analyzed in detail in Ref. [31] and here; we see no large-scale avalanches below yielding, whereas we find system-spanning avalanches above. Although we do not compute a correlation length or size of correlated events as in Refs. [24,25], the observations reported in Refs. [24,25] below yielding are puzzling since, in the steady state, there are in fact very few plastic rearrangements. A possible resolution is that below yielding, in the transient regime, rearrangement events take place that are larger and relax more slowly for strain amplitudes that are closer to (but smaller than) the yielding amplitude. Thus, for any fixed observation time, one would find a larger size of rearrangements for cyclic deformations with amplitude closer to the yield amplitude. We illustrate this in Fig. 7, where we show the energy drops during avalanches as a function of cycles of deformation.

Experimental results for uniformly sheared colloidal glasses [60] have been interpreted in terms of coupling of strain to concentration (or density). But direct evidence for such coupling is lacking. In our work, we observe that strain localization is accompanied by a reduction in the local density and thus supports the possibility of such a coupling playing a role in yielding. This observation needs to be explored further in order to make further contact with the analysis mentioned above. An attempt was made in

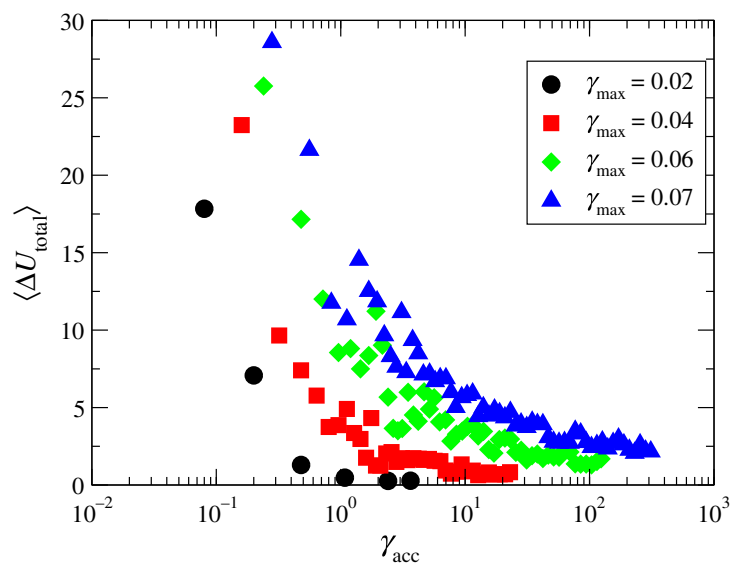

FIG. 7. The total energy drop during a plastic rearrangement (for events in the first quadrant, with strain values increasing from 0 to $\gamma_{\max }$ ) is computed for energy minima obtained at $T=1.0$, $N=64000$, as a way of characterizing the size of rearrangement events, as a function of the accumulated strain, for a range of strain amplitudes. The results show that the rearrangement events relax to their asymptotic values more slowly as the yield strain amplitude is approached, and at any value of the accumulated strain, they are larger for strain amplitudes that are closer to the yielding strain amplitude. 
Ref. [48], using computer simulations, to correlate the presence of shear banding with the spatial variation of structure in the sheared systems. The results indicate an anticorrelation with local strain rates with the prevalence of well-packed structure, which is entirely consistent with findings here.

It has been discussed in interpreting experimental results concerning soft glassy materials (see, e.g., Refs. $[4,61])$ that shear banding in glasses involves a competition between aging and rejuvenation. But direct evidence, experimental or otherwise, has not been available so far. To our knowledge, our work offers the first concrete evidence in this regard. A number of model calculations and simulations [51,62-66] have sought to understand strain localization in terms of a competition between rates of relaxation or aging, and rejuvenation arising from flow. These studies, mostly in the context of uniform shear (but also cyclic shear [51]), implicitly or explicitly assume that the consequence of shear deformation is the rejuvenation of the glasses, whereas aging may arise from thermally induced, activated relaxation. While these effects may have validity up to a point, a key observation arising from our work is that shear deformation alone leads to annealing, both below and above yielding. This is strikingly so for cyclic deformation, but our observations also have implications for annealing effects under uniform deformation. Thus, our results suggest that the nature of the aging and rejuvenation mechanisms in modeling shear banding should be examined (if not at a phenomenological level, certainly with regard to investigating microscopic origins) keeping in mind that shear deformation can not only lead to rejuvenation but also annealing. In comparing our results with other investigations, however, a distinction that needs to be kept in mind is that the persistent shear banding that is analyzed in most of the mentioned studies is the saturation of the width of the shear band to a finite value as shear strain increases arbitrarily, under flow conditions. In contrast, the stable shear bands that we observe are at a fixed strain amplitude, and their widths do increase as the strain amplitude is increased. The present results do not permit us to address whether the shear bands we observe will grow to arbitrarily large widths as the strain amplitude increases to large values [66]. Analyzing this question, along with the role of finite temperatures and shear rates, is an obvious, important direction for future work.

\section{ACKNOWLEDGMENTS}

We wish to thank Pinaki Chaudhuri, Michael Falk, Giuseppe Foffi, Jürgen Horbach, Smarajit Karmakar Kristen Martens, Itamar Procaccia, Francesco Sciortino, and Vishwas V. Vasisht for useful discussions. We gratefully acknowledge Thematic Unit of Excellence on Computational Materials Science and Sheikh Saqr Laboratory, JNCASR, Bengaluru, and CRAY for computational resources and support. We especially wish to thank Sudhakar Yerneni and Raviteja Kuppili for substantial support with CRAY computational facility.

[1] C. A. Schuh, T. C. Hufnagel, and U. Ramamurty, Mechanical Behavior of Amorphous Alloys, Acta Mater. 55, 4067 (2007).

[2] P. Schall and M. van Hecke, Shear Bands in Matter with Granularity, Annu. Rev. Fluid Mech. 42 (2010).

[3] M. L. Falk and J. S. Langer, Deformation and Failure of Amorphous, Solidlike Materials, Annu. Rev. Condens. Matter Phys. 2, 353 (2011).

[4] D. Bonn, M. M. Denn, L. Berthier, T. Divoux, and S. Manneville, Yield Stress Materials in Soft Condensed Matter, Rev. Mod. Phys. 89, 035005 (2017).

[5] A. Nicolas, E. E. Ferrero, K. Martens, and J.-L. Barrat, Deformation and Flow of Amorphous Solids: A Review of Mesoscale Elastoplastic Models, Rev. Mod. Phys. 90, 045006 (2018).

[6] F. M. Chester and J. S. Chester, Ultracataclasite Structure and Friction Processes of the Punchbowl Fault, San Andreas System, California, Tectonophysics 295, 199 (1998).

[7] E. Pekarskaya, C. P. Kim, and W. L. Johnson, In Situ Transmission Electron Microscopy Studies of Shear Bands in a Bulk Metallic Glass Based Composite, J. Mater. Res. 16, 2513 (2001).

[8] I. Cohen, B. Davidovitch, A. B. Schofield, M. P. Brenner, and D. A. Weitz, Slip, Yield, and Bands in Colloidal Crystals under Oscillatory Shear, Phys. Rev. Lett. 97, 215502 (2006).

[9] J. T. Uhl et al., Universal Quake Statistics: From Compressed Nanocrystals to Earthquakes, Sci. Rep. 5, 16493 (2015).

[10] P. Hébraud and F. Lequeux, Mode-Coupling Theory for the Pasty Rheology of Soft Glassy Materials, Phys. Rev. Lett. 81, 2934 (1998).

[11] R. G. Larson, The Structure and Rheology of Complex Fluids (Oxford University Press, New York, Oxford, 1999).

[12] J.D. Ferry and J.D. Ferry, Viscoelastic Properties of Polymers (John Wiley \& Sons, New York, 1980).

[13] Y. Shi and M. L. Falk, Strain Localization and Percolation of Stable Structure in Amorphous Solids, Phys. Rev. Lett. 95, 095502 (2005).

[14] D. J. Pine, J. P. Gollub, J. F. Brady, and A. M. Leshansky, Chaos and Threshold for Irreversibility in Sheared Suspensions, Nature (London) 438, 997 (2005).

[15] C. E. Maloney and A. Lemaître, Amorphous Systems in Athermal, Quasistatic Shear, Phys. Rev. E 74, 016118 (2006).

[16] L. Corte, P. M. Chaikin, J. P. Gollub, and D. J. Pine, Random Organization in Periodically Driven Systems, Nat. Phys. 4, 420 (2008).

[17] K. A. Dahmen, Y. Ben-Zion, and J. T. Uhl, Micromechanical Model for Deformation in Solids with Universal Predictions for Stress-Strain Curves and Slip Avalanches, Phys. Rev. Lett. 102, 175501 (2009). 
[18] M. L. Manning, E. G. Daub, J. S. Langer, and J. M. Carlson, Rate-Dependent Shear Bands in a Shear-TransformationZone Model of Amorphous Solids, Phys. Rev. E 79, 016110 (2009).

[19] S. Karmakar, E. Lerner, and I. Procaccia, Statistical Physics of the Yielding Transition in Amorphous Solids, Phys. Rev. E 82, 055103(R) (2010).

[20] J. L. Barrat and A. Lemaître, Heterogeneities in Amorphous Systems under Shear, edited by L. Berthier, G. Biroli, J. P. Bouchaud, L. Cipelletti, and W. v. Saarloos, Dynamical Heterogeneities in Glasses, Colloids, and Granular Media (Oxford Science Publications, Oxford, 2011), Chapter 8, pp. 264-297.

[21] R. Dasgupta, H. G. E. Hentschel, and I. Procaccia, Microscopic Mechanism of Shear Bands in Amorphous Solids, Phys. Rev. Lett. 109, 255502 (2012).

[22] N. C. Keim and P. E. Arratia, Yielding and Microstructure in a 2D Jammed Material under Shear Deformation, Soft Matter 9, 6222 (2013).

[23] J. Lin, E. Lerner, A. Rosso, and M. Wyart, Scaling Description of the Yielding Transition in Soft Amorphous Solids at Zero Temperature, Proc. Natl. Acad. Sci. U.S.A. 111, 14382 (2014).

[24] E. D. Knowlton, D. J. Pine, and L. Cipelletti, A Microscopic View of the Yielding Transition in Concentrated Emulsions, Soft Matter 10, 6931 (2014).

[25] K. H. Nagamanasa, S. Gokhale, A. K. Sood, and R. Ganapathy, Experimental Signatures of a Nonequilibrium Phase Transition Governing the Yielding of a Soft Glass, Phys. Rev. E 89, 062308 (2014).

[26] D. V. Denisov, M. T. Dang, B. Struth, A. Zaccone, G. H. Wegdam, and P. Schall, Sharp Symmetry-Change Marks the Mechanical Failure Transition of Glasses, Sci. Rep. 5, 14359 (2015).

[27] I. Regev, J. Weber, C. Reichhardt, K. A. Dahmen, and T. Lookman, Reversibility and Criticality in Amorphous Solids, Nat. Commun. 6, 8805 (2015).

[28] C. Liu, E. E. Ferrero, F. Puosi, J.-L. Barrat, and K. Martens, Driving Rate Dependence of Avalanche Statistics and Shapes at the Yielding Transition, Phys. Rev. Lett. 116, 065501 (2016).

[29] P. K. Jaiswal, I. Procaccia, C. Rainone, and M. Singh, Mechanical Yield in Amorphous Solids: A First-Order Phase Transition, Phys. Rev. Lett. 116, 085501 (2016).

[30] G. P. Shrivastav, P. Chaudhuri, and J. Horbach, Yielding of Glass under Shear: A Directed Percolation Transition Precedes Shear-Band Formation, Phys. Rev. E 94, 042605 (2016).

[31] P. Leishangthem, A. D. S. Parmar, and S. Sastry, The Yielding Transition in Amorphous Solids under Oscillatory Shear Deformation, Nat. Commun. 8, 14653 (2017).

[32] G. Parisi, I. Procaccia, C. Rainone, and M. Singh, Shear Bands as Manifestation of a Criticality in Yielding Amorphous Solids, Proc. Natl. Acad. Sci. U.S.A. 114, 5577 (2017).

[33] P. Urbani and F. Zamponi, Shear Yielding and Shear Jamming of Dense Hard Sphere Glasses, Phys. Rev. Lett. 118, 038001 (2017).

[34] Y. Jin, P. Urbani, F. Zamponi, and H. Yoshino, A StabilityReversibility Map Unifies Elasticity, Plasticity, Yielding and
Jamming in Hard Sphere Glasses, Sci. Adv. 4, eaat6387 (2018).

[35] M. Ozawa, L. Berthier, G. Biroli, A. Rosso, and G. Tarjus, Random Critical Point Separates Brittle and Ductile Yielding Transitions in Amorphous Materials, Proc. Natl. Acad. Sci. U.S.A. 115, 6656 (2018).

[36] M. Popović, T. W. J. de Geus, and M. Wyart, Elastoplastic Description of Sudden Failure in Athermal Amorphous Materials During Quasistatic Loading, Phys. Rev. E 98, 040901(R) (2018).

[37] A. S. Argon, Plastic Deformation in Metallic Glasses, Acta Metall. 27, 47 (1979).

[38] M. L. Falk and J. S. Langer, Dynamics of Viscoplastic Deformation in Amorphous Solids, Phys. Rev. E 57, 7192 (1998).

[39] G. Picard, A. Ajdari, F. Lequeux, and L. Bocquet, Elastic Consequences of a Single Plastic Event: A Step Towards the Microscopic Modeling of the Flow of Yield Stress Fluids, Eur. Phys. J. E 15, 371 (2004).

[40] F. Puosi, J. Rottler, and J.-L. Barrat, Time-Dependent Elastic Response to a Local Shear Transformation in Amorphous Solids, Phys. Rev. E 89, 042302 (2014).

[41] M. Talamali, V. Petäjä, D. Vandembroucq, and S. Roux, Strain Localization and Anisotropic Correlations in a Mesoscopic Model of Amorphous Plasticity, C.R. Mec. 340, 275 (2012).

[42] B. Tyukodi, S. Patinet, S. Roux, and D. Vandembroucq, From Depinning Transition to Plastic Yielding of Amorphous Media: A Soft-Modes Perspective, Phys. Rev. E 93, 063005 (2016).

[43] V. Lubchenko and P. G. Wolynes, Barrier Softening Near the Onset of Nonactivated Transport in Supercooled Liquids: Implications for Establishing Detailed Connection between Thermodynamic and Kinetic Anomalies in Supercooled Liquids, J. Chem. Phys. 119, 9088 (2003).

[44] V. Lubchenko and P. G. Wolynes, Theory of Aging in Structural Glasses, J. Chem. Phys. 121, 2852 (2004).

[45] V. Lubchenko, Shear Thinning in Deeply Supercooled Melts, Proc. Natl. Acad. Sci. U.S.A. 106, 11506 (2009).

[46] A. Wisitsorasak and P. G. Wolynes, On the Strength of Glasses, Proc. Natl. Acad. Sci. U.S.A. 109, 16068 (2012).

[47] S. Saw, S. Abraham, and P. Harrowell, Nonaffine Displacements and the Nonlinear Response of a Strained Amorphous Solid, Phys. Rev. E 94, 022606 (2016).

[48] V. V. Vasisht, G. Roberts, and E. Del Gado, Emergence and Persistence of Flow Inhomogeneities in the Yielding and Fluidization of Dense Soft Solids, arXiv:1709.08717.

[49] D. Fiocco, G. Foffi, and S. Sastry, Oscillatory Athermal Quasistatic Deformation of a Model Glass, Phys. Rev. E 88, 020301(R) (2013).

[50] D. Fiocco, G. Foffi, and S. Sastry, Encoding of Memory in Sheared Amorphous Solids, Phys. Rev. Lett. 112, 025702 (2014).

[51] R. Radhakrishnan and S. M. Fielding, Shear Banding of Soft Glassy Materials in Large Amplitude Oscillatory Shear, Phys. Rev. Lett. 117, 188001 (2016).

[52] T. Kawasaki and L. Berthier, Macroscopic Yielding in Jammed Solids Is Accompanied by a Nonequilibrium First-Order Transition in Particle Trajectories, Phys. Rev. E 94, 022615 (2016). 
[53] Nikolai V. Priezjev, The Yielding Transition in Periodically Sheared Binary Glasses at Finite Temperature, Comput. Mater. Sci., 150, 162 (2018).

[54] K. M. Salerno and M. O. Robbins, Effect of Inertia on Sheared Disordered Solids: Critical Scaling of Avalanches in Two and Three Dimensions, Phys. Rev. E 88, 062206 (2013).

[55] D. J. Evans and G. P. Morriss, Nonlinear-Response Theory for Steady Planar Couette Flow, Phys. Rev. A 30, 1528 (1984).

[56] S. Plimpton, Fast Parallel Algorithms for Short-Range Molecular Dynamics, J. Comput. Phys. 117, 1 (1995).

[57] See Supplemental Material at http://link.aps.org/ supplemental/10.1103/PhysRevX.9.021018 for two visualizations have been shown for two different cases: (a) An initially homogeneous glass that shear bands when subjected to cyclic deformation, and (b) An initially shear banded glass in which the shear band heals when cyclically deformed below the yielding strain amplitude. The visualization "from-liq-074.mp4" demonstrates shear-band formation for an initially homogeneous sample obtain by quenching a liquid from a high temperature $T=1$, stroboscopically over repeated cycles of strain for the strain amplitude $\gamma_{\max }=0.074$ ( $>\gamma_{y}$, the yield strain amplitude). The visualization "From08-at-070.mp4" shows the healing of a shear banded sample, which is subjected to repeated cycles of strain at strain amplitude $\gamma_{\max }=0.070$, just below the yield strain amplitude. The color of particles corresponds to the meansquared displacement between two successive cycles, as explained in the main text..

[58] D. J. Lacks and M. J. Osborne, Energy Landscape Picture of Overaging and Rejuvenation in a Sheared Glass, Phys. Rev. Lett. 93, 255501 (2004).

[59] N. C. Keim and P. E. Arratia, Yielding and Microstructure in a 2D Jammed Material under Shear Deformation, Soft Matter 9, 6222 (2013).

[60] R. Besseling, L. Isa, P. Ballesta, G. Petekidis, M. E. Cates, and W. C. K. Poon, Shear Banding and Flow-Concentration Coupling in Colloidal Glasses, Phys. Rev. Lett. 105, 268301 (2010).

[61] P. C. F. Møller, S. Rodts, M. A. J. Michels, and D. Bonn, Shear Banding and Yield Stress in Soft Glassy Materials, Phys. Rev. E 77, 041507 (2008).

[62] E. A. Jagla, Strain Localization Driven by Structural Relaxation in Sheared Amorphous Solids, Phys. Rev. E 76, 046119 (2007).

[63] P. Coussot and G. Ovarlez, Physical Origin of ShearBanding in Jammed Systems, Eur. Phys. J. E 33, 183 (2010).

[64] E. A. Jagla, Shear Band Dynamics from a Mesoscopic Modeling of Plasticity, J. Stat. Mech. (2010) P12025.

[65] K. Martens, L. Bocquet, and J.-L. Barrat, Spontaneous Formation of Permanent Shear Bands in a Mesoscopic Model of Flowing Disordered Matter, Soft Matter 8, 4197 (2012).

[66] D. D. Alix-Williams and M. L. Falk, Shear Band Broadening in Simulated Glasses, Phys. Rev. E 98, 053002 (2018). 